\title{
Investigation of the role of miR-221 in diabetic peripheral neuropathy and related molecular mechanisms
}

\author{
Xiaole Wu ${ }^{1,2, A-F}$, Xiaoyu Wang ${ }^{3, B, C, E, F}$, Yiyu Yin ${ }^{4, B, C, E, F}$, Lei Zhu ${ }^{5, B, C, E, F}$, Fengchao Zhang ${ }^{2, B, C, E, F}$, Jianping Yang ${ }^{1, C, E, F}$ \\ 1 Department of Anesthesiology, The First Affiliated Hospital of Soochow University, Suzhou, China \\ 2 Department of Anesthesiology, Xuzhou Children's Hospital, Xuzhou Medical University, China \\ ${ }^{3}$ Department of Thoracic Surgery, Xuzhou Children's Hospital, Xuzhou Medical University, China \\ ${ }^{4}$ Department of General Surgery, Xuzhou Children's Hospital, Xuzhou Medical University, China \\ ${ }^{5}$ Intensive Care Unit, Xuzhou Children's Hospital, Xuzhou Medical University, China \\ A - research concept and design; $\mathrm{B}$ - collection and/or assembly of data; C - data analysis and interpretation; \\ $D$ - writing the article; $E$ - critical revision of the article; $F$ - final approval of the article
}

\author{
Address for correspondence \\ Jianping Yang \\ E-mail: hejunqin_1@sohu.com \\ Funding sources \\ None declared \\ Conflict of interest \\ None declared

\section{Acknowledgements} \\ This work was non-financially supported \\ by Xuzhou Children's Hospital, Xuzhou Medical \\ University, and The First Affiliated Hospital \\ of Soochow University, Suzhou, China.
}

Received on July 10,2020

Reviewed on July 15, 2020

Accepted on December 6, 2020

Published online on May 20, 2021

Cite as

Wu X, Wang X, Yin Y, Zhu L, Zhang F, Yang J. Investigation of the role of miR-221 in diabetic peripheral neuropathy and related molecular mechanisms. Adv Clin Exp Med. 2021;30(6):623-632. doi:10.17219/acem/131217

DOI

10.17219/acem/131217

\section{Copyright}

Copyright by Author(s)

This is an article distributed under the terms of the

Creative Commons Attribution 3.0 Unported (CC BY 3.0)

(https://creativecommons.org/licenses/by/3.0/)

\section{Abstract}

Background. Diabetic peripheral neuropathy (DPN) is one of the most common complications of diabetes, but the molecular mechanisms of DPN are still unclear.

Objectives. To investigate the role of miR-221 in DPN and the related molecular mechanisms.

Materials and methods. Streptozotocin (STZ) was used to establish an in vivo DPN model. An in vitro DPN model was established using high glucose-induced SH-SY5Y cells. The pain condition of rats was measured by evaluating the 50\% paw withdrawal threshold (PWT) and paw withdrawal latency (PWL). Serum exosomes were extracted and identified. Expression of miR-221 in serum exosomes and serum SOCS3 expression were determined using reverse-transcription quantitative polymerase chain reaction (RT-qPCR). Western blotting was used to measure the protein levels of SOCS3, bradykinin (BK) and prostaglandin E2 (PEG2). The dual luciferase reporter assay was performed to confirm SOCS3 3'-UTR as a target of miR-221. The serum or cell supernatant levels of PEG2, BK, interleukin (IL)-6, IL-1B, and tumor necrosis factor alpha (TNF-a) were measured using enzyme-linked immunosorbent assay (ELISA).

Results. Induction of the lenti-miR-221 inhibitor significantly decreased the expression of miR-221 in DPN rats. Both 50\% PWT and PWL values were markedly decreased in DPN rats. When miR-221 was inhibited, the 50\% PWT and PWL values were both significantly increased. Knockdown of miR-221 significantly increased the expression of SOCS3 and decreased the expression of NF-KB. Furthermore, knockdown of miR-221 remarkably decreased the expression of PEG2, BK, IL-6, IL-1B, and TNF-a in both STZ-treated DPN rats and high glucose-induced SH-SY5Y cells, which was reversed by inhibition of SOCS3. The dual luciferase reporter assay showed that miR-221 directly targeted and negatively regulated SOCS3.

Conclusions. Inhibition of miR-221 can reduce pain and decrease expression of inflammatory factors through targeting SOCS3 in DPN.

Key words: exosomes, miR-221, SOCS3, diabetic peripheral neuralgia 


\section{Background}

Diabetic peripheral neuropathy (DPN) is one of the most common complications of diabetes. Almost 50\% of diabetic patients develop DPN during their lifetime and about $10-26 \%$ of newly diagnosed diabetic patients suffer from DPN. ${ }^{1,2}$ Compared to other types of peripheral neuropathy, the development of DPN is more rapid. Research has reported that DPN is the primary cause of diabetic foot ulcer and amputation, which lead to disability. ${ }^{3-5}$ Generally, it is considered that DPN is the result of many factors related to the hyperglycemic condition. Many factors influence DPN incidence, including glycosylation of tissue protein, oxidative stress, mitochondrial damage, inflammation activation, and activation of the polyol pathway. ${ }^{6-8}$ However, the molecular mechanisms of DPN are still unclear.

MicroRNA (miRNA) is a kind of endogenous non-coding RNA with a length of about 20-24 bases that plays wide roles in organisms. ${ }^{9}$ Among miRNAs, miR-221 plays important roles in many diseases. Oh et al. found that miR-221 could inhibit oxidative stress through regulating DJ-1 in Parkinson's disease..$^{10}$ Fornari et al. demonstrated that $m i R-221$ could induce sorafenib resistance by inhibiting caspase-3-mediated apoptosis in hepatoma cells. ${ }^{11}$ In addition, $m i R-221$ was found to be upregulated in diabetes and promote diabetes development. ${ }^{12}$ However, the role of $m i R-221$ in DPN has not been elucidated yet.

\section{Objectives}

In the present study, we aimed to investigate the role of $m i R-221$ in DPN. We demonstrated that inhibition of $m i R-221$ led to reduction of pain and decreased inflammatory factors through targeting SOCS3 in the DPN model. This research might give deeper insights into the molecular mechanisms of DPN.

\section{Materials and methods}

\section{Animals and treatment}

For establishment of the DPN in vivo model, 40 male Sprague Dawley (SD) rats (215 \pm 15 g, 3-month old) were purchased from the Laboratory Animal Center of Xuzhou Medical University, China. The rats were kept in microisolator cages in a light-controlled room under a $12 \mathrm{~h} / 12 \mathrm{~h}$ light/dark cycle and a controlled temperature $\left(23-25^{\circ} \mathrm{C}\right)$, and had free access to food and water. All efforts were made to avoid unnecessary pain suffered by the animals. This study was approved by the Institutional Animal Care Committee at Xuzhou Children's Hospital.

Animals were divided into 4 groups ( $\mathrm{n}=10$ per group): 1) control group; 2) streptozotocin (STZ)-induced DPN group; 3) DPN and miR-221 inhibitor group; and 4) DPN and inhibitor negative control (NC) group. For establishment of the DPN model, rats received daily intraperitoneal injection of STZ (Sinopharm Group, Co. Ltd., Shanghai, China) at a dose of $60 \mathrm{mg} / \mathrm{kg}$. After 7 days of injection, the blood glucose level of the rats was evaluated using a blood glucose meter (Abbott Laboratories, Chicago, USA), and a blood glucose level $>16.7 \mathrm{~mol} / \mathrm{L}$ was regarded as successful establishment of the diabetes model. After 14 days of injection, a von Frey fiber pain meter (Stoelting, Wood Dale, USA) was used for measurement of the $50 \%$ paw withdrawal threshold (PWT), and 50\% PWT $<4$ g was regarded as successful establishment of the DPN model. The rats in the control group received daily injections of normal saline with the same volume.

For the DPN and miR-221 inhibitor or DPN and inhibitor NC group, the $m i R-221$ inhibitor was designed and synthesized by GeneChem Corp. (Shanghai, China). The sequence of the $m i R-221$ inhibitor was: 5'-GAAACCCAGCAGACAAUGUAGCU-3'. The construction and packaging of the lentivirus of the $m i R-221$ inhibitor (lenti-miR-221-inhibitor) and lenti-miR-NC were accomplished by GeneChem Corp. Briefly, miRNA oligos with the $m i R-221$ inhibitor/NC sequence were synthesized and subcloned into the pcDNA6.2 $2^{\mathrm{TM}}$ GW/EmGFP-miR vector (Invitrogen, Carlsbad, USA). The lenti-miR-221-inhibitor/NC vector was obtained after att $\mathrm{B}$ and att $\mathrm{P}$ mediated recombination (BP reaction) between the miRNA vector and pDONR221 vector, and att $L$ and att $R$ mediated recombination (LR reaction) between the entry vectors and pLenti6.3/V5-dest. The lentimiR-221-inhibitor/ $\mathrm{NC}$ vector was then packaged with active titer $2 \times 10^{8} \mathrm{TU} / \mathrm{mL}$. The rats the in DPN and $m i R-221$ inhibitor/NC group were DNP rats who received tail vein injection of $100 \mu \mathrm{L}$ lenti-miR-221-inhibitor/NC vectors $\left(2 \times 10^{8} \mathrm{TU} / \mathrm{mL}\right)$. Treatment was conducted immediately after injection of STZ. All experiments were repeated in triplicate.

\section{Measurement of PWT and PWL}

The PWT was measured using a von Frey fiber pain meter (Stoelting). Briefly, rats were placed in a transparent plexiglas box with a hole of $0.5 \times 0.5 \mathrm{~cm}^{2}$ in the bottom. The plantar of the rats was stimulated by gradually increasing the buckling force from $0.57 \mathrm{~g}$. The force of the leg retraction reaction was regarded as the PWT and 50\% PWT was calculated. Each animal was evaluated 5 times. The paw withdrawal latency (PWL) was measured using an automatic thermal pain stimulator (BMC-410C; Institute of Biomedical Engineering, Chinese Academy of Medical Sciences, Beijing, China). Briefly, rats were put on a glass plate and exposed to thermal radiation. The time from the beginning of irradiation to the emergence of leg raising avoidance was regarded as the PWL. Each animal was evaluated 5 times. 


\section{Extraction and identification of serum exosomes}

Blood samples were obtained from the rats after 14 days of treatment. Samples were centrifuged at $3000 \times \mathrm{g}$ at $4^{\circ} \mathrm{C}$ for $5 \mathrm{~min}$. The serum exosomes were extracted using ExoQuick Exosome Precipitation Solution (ExoQuick; System Biosciences, Palo Alto, USA) in strict adherence with the manufacturer's instructions. For identification of the extracted exosomes, a flow cytometer (Beckman Coulter, Brea, USA) was used for measurement of the surface biomarkers CD63, CD90, CD45, and CD34. Briefly, acetaldehyde/sulfate latex beads $(4 \mu \mathrm{M})$ were added into $30 \mu \mathrm{g}$ extracted exosomes to achieve $100 \mu \mathrm{L}$. After adding $1 \mathrm{~mL}$ of phosphate-buffered saline (PBS) and incubation for $2 \mathrm{~h}$, glycine (100 mmol) was added and the samples were incubated with primary antibodies of anti-CD63 (ab108950; Abcam, Cambridge, USA), anti-CD90 (ab226; Abcam), anti-CD45 (ab10558; Abcam), and anti-CD34 (ab81289; Abcam) at $4^{\circ} \mathrm{C}$ overnight. After incubation with the corresponding secondary antibody Goat Anti-Rabbit IgG H\&L (horseradish peroxidase (HRP); ab205718; Abcam) at $37^{\circ} \mathrm{C}$ for $30 \mathrm{~min}$, the biomarkers were analyzed using flow cytometry (FCM).

For observation of the morphology of the extracted exosomes, the exosomes were also observed using a transmission electron microscope (Hitachi, Tokyo, Japan).

\section{Cell culture and transfection}

For the in vitro DPN model, the SH-SY5Y cell line from American Type Cell Culture (ATCC, Manassas, USA) was treated with $50 \mathrm{mmol} / \mathrm{L}$ of D-glucose (Sigma-Aldrich, St. Louis, USA). Cells were cultured in Dulbecco's modified Eagle's medium (DMEM; Gibco, Gaithersburg, USA) with $10 \%$ fetal bovine serum (FBS; Gibco), $100 \mathrm{IU} / \mathrm{mL}$ of penicillin, and $100 \mu \mathrm{g} / \mathrm{mL}$ of streptomycin at $37^{\circ} \mathrm{C}$ with $5 \% \mathrm{CO}_{2}$. Normal cells were treated with $5 \mathrm{mmol} / \mathrm{L} \mathrm{D}$-glucose.

For cell transfection, cells were transfected with $m i R-221$ inhibitor/mimics or inhibitor/mimics $\mathrm{NC}$, as well as siSOCS3 or si-NC (all $5 \mathrm{nM}$ ) using Lipofectamine 3000 (Invitrogen, Carlsbad, USA) in serum-free Opti-MEM medium (Gibco) according to the manufacturer's instructions. The $m i R-221$ mimics/inhibitor, mimics/inhibitor $\mathrm{NC}$, si-SOCS3, and si-NC were designed and synthesized by GeneChem Corp.

\section{RT-qPCR}

Total SOCS3 RNA was extracted from the serum samples or cells using TRIzol reagent (Thermo Fisher Scientific, Waltham, USA). The extraction of $m i R-221$ was performed using a mirVana miRNA isolation kit (Ambion, Austin, USA) strictly according to the manufacturer's instructions. RNA concentration was determined using a NanoDrop ND-1000 spectrophotometer (NanoDrop Technologies,
Wilmington, USA). The TaqMan ${ }^{\circledR}$ RNA Reverse Transcription Kit (Applied Biosystems, Foster City, USA) was used to convert RNA into cDNA. The TaqMan ${ }^{\circledR}$ MicroRNA Reverse Transcription Kit (Applied Biosystems) was used for reverse transcription of miRNA. The SYBR Premix Ex Taq ${ }^{\mathrm{TM}}$ II Kit (Solarbio Science \& Technology Co. Ltd., Beijing, China) was used for reverse-transcription quantitative polymerase chain reaction (RT-qPCR) in an ABI StepOnePlus system (Applied Biosystems). The thermocycling conditions were as follows: initial activation step at $95^{\circ} \mathrm{C}$ for $15 \mathrm{~min}, 40$ cycles, denaturation at $94^{\circ} \mathrm{C}$ for $15 \mathrm{~s}$, annealing at $55^{\circ} \mathrm{C}$ for $30 \mathrm{~s}$, and extension at $72^{\circ} \mathrm{C}$ for $30 \mathrm{~s}$. Primers are listed Table 1 . The relative RNA levels were calculated using the $2^{-\triangle \triangle \mathrm{Cq}}$ method. U6 and GAPDH were used as internal controls for miR-221 and SOCS3, respectively.

Table 1. Sequences used in PCR

\begin{tabular}{|l|c|}
\multicolumn{1}{|c|}{ Senes } & Sequence \\
\hline miR-221 & F 5'-CAGCATACATGATTCCTTGTGA-3' \\
R 5'-CTTTGGTGTTTGAGATGTTTGG-3' \\
SOCS3 & F 5'-CCTGCGCCTCAAGACCTTC-3' \\
R 5'-GTCACTGCGCTCCAGTAGAA-3' & F 5'-AGCGAGCATCCCCCAAAGTT-3' \\
GAPDH & R 5'-GGGCACGAGGCTCATCATT-3' \\
U6 & F 5'-TGCGGGTGCTCGCTTCGGCAGC-3' \\
& R 5'-CCAGTGCAGGGTCCGAGGT-3' \\
\hline
\end{tabular}

\section{Western blotting}

Western blotting was used to measure the protein levels of SOCS3, bradykinin (BK) and prostaglandin E2 (PEG2). Proteins were extracted from serum samples or cells using radioimmunoprecipitation assay (RIPA) buffer (Vazyme Biotec Co. Ltd., Nanjing, China) and the concentrations were determined using a BCA assay kit (Sigma-Aldrich). After being subjected to $10 \%$ sodium dodecyl sulfate polyacrylamide gel electrophoresis (SDS-PAGE) and transferred to polyvinylidene difluoride (PVDF) film, samples were blocked using non-fat milk at room temperature for $1 \mathrm{~h}$. The same samples were then incubated with primary antibodies of anti-SOCS3 (ab16030; Abcam), PEG2 (ab2318; Abcam) and BK (ab47864, Abcam) at $4^{\circ} \mathrm{C}$ overnight followed by incubation with the corresponding secondary antibody (Goat Anti-Rabbit IgG H\&L, ab205718; Abcam). The films were scanned using the Pierce ECL Western Blotting Substrate (Pierce, Shanghai, China) and analyzed using a Bio-Rad gel imaging system (Bio-Rad, Hercules, USA).

\section{Dual luciferase reporter assay}

The dual luciferase reporter assay was performed to confirm the SOCS3 3'-UTR as a target of miR-221. Briefly, the wild-type (WT) SOCS3 3'-UTR or mutant (Mut) type 
was amplified and sub-cloned into a pGL4.10 luciferase reporter vector and the cells were transfected with miR-221 mimics, inhibitor or mimics/inhibitor NC using Lipofectamine 3000 (Invitrogen). Luciferase assays were performed using a Bright-Glo ${ }^{\text {TM }}$ Luciferase Assay System (Promega, Madison, USA) after $48 \mathrm{~h}$ of transfection and the luciferase activity was normalized to the values of Renilla luciferase activity.

\section{Measurement of PEG2, BK, IL-6, IL-1 $\beta$, and TNF-a}

Serum or cell supernatant levels of PEG2, BK, interleukin (IL) -6 , IL-1 $\beta$, and tumor necrosis factor alpha (TNF- $\alpha$ ) were measured with enzyme-linked immunosorbent assay (ELISA) according to the manufacturer's instructions using commercial kits: Prostaglandin E2 ELISA Kit (Abcam); Bradykinin ELISA Kit (Abcam); Interleukin 6 (Sandwich ELISA) ELISA Kit (LSBio, Inc.); Interleukin $1 \beta$ (Sandwich ELISA) ELISA Kit (LSBio, Inc.); and TNF- $\alpha$ ELISA Kit (LSBio, Inc.).

\section{Statistical analyses}

Data are expressed as the mean \pm standard deviation (SD). Comparisons were conducted using one-way analysis of variance (ANOVA) followed by the Tukey's post hoc test. Findings were considered to be statistically significant when the p-value was less than 0.05. All analyses were performed using SPSS v. 18.0 (SPSS Inc., Chicago, USA).

\section{Results}

\section{MiR-221 was upregulated in serum-extracted exosomes in STZ-induced DPN rats}

First, the exosomes extracted from the serum taken from the rats were identified. The morphology of the exosomes was photographed and the mean diameter was about $70 \mathrm{~nm}$ (Fig. 1A,B). Flow cytometry and western blotting results showed that CD63 and CD90 were positively expressed, while CD45 and CD34 were negatively expressed in the exosomes (Fig. 1C,D), indicating the successful extraction of the exosomes. Next, the expression of $m i R-221$ was determined using RT-qPCR. It was observed that miR-221 was markedly upregulated in the serum exosomes of the DPN rats (Fig. 1E), suggesting that $m i R-221$ is abnormally expressed and might play a role in DPN.

\section{Inhibition of miR-221 reduced the pain condition of STZ-induced DPN rats}

To investigate the role of $m i R-221$ in DPN, we injected the lenti-miR-221 inhibitor into the rats and observed alterations in their pain condition. As shown in Fig. 2A, the blood glucose level increased remarkably in DPN rats compared with controls $(\mathrm{p}<0.05)$. Induction of the lentimiR-221 inhibitor significantly decreased the expression of $m i R-221$ in DPN rats compared with the NC group (p < 0.05, Fig. 2B). Both 50\% PWT and PWL values were markedly decreased in DPN rats compared with controls
A

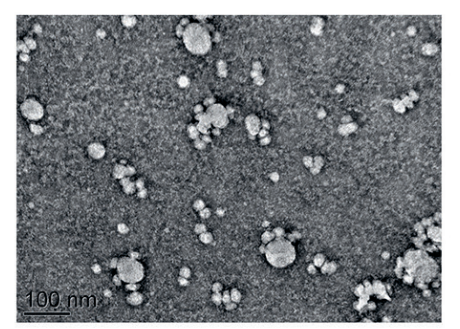

C
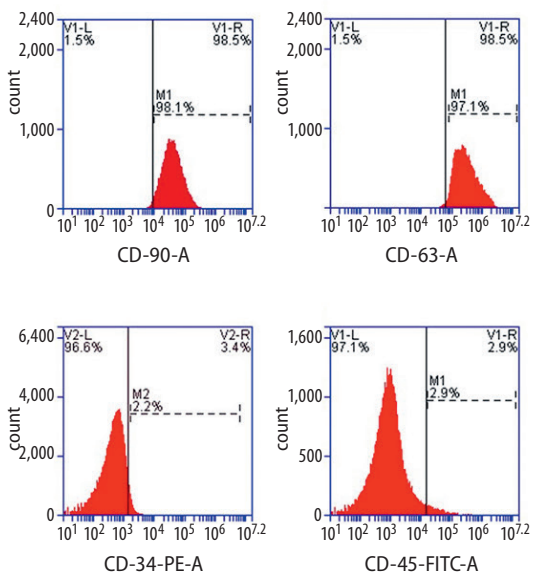

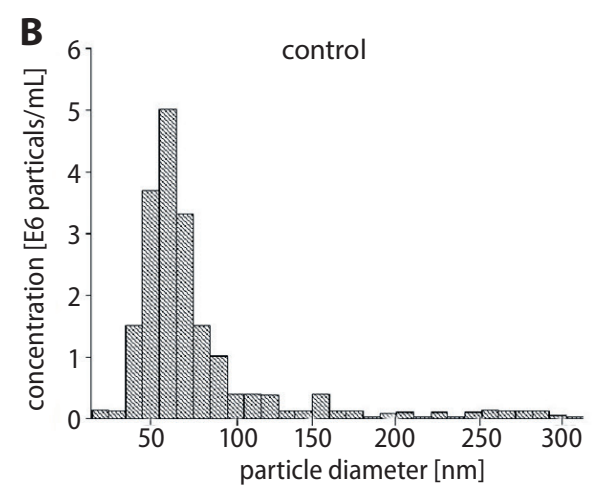

D

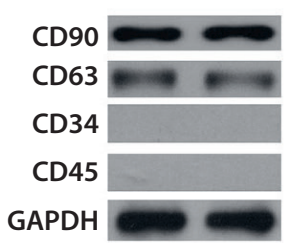

E

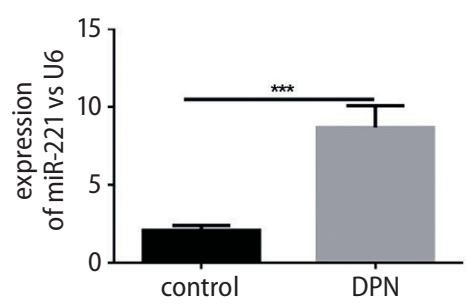

Fig. 1. MiR-221 was upregulated in serumextracted exosomes from STZ-induced DPN rats

A. Morphology of serumextracted exosomes under TEM (100 nm); B. Distribution of the diameters of exosomes; C. FCM results for surface biomarkers of CD90, CD63, CD34, and CD45; D. Protein levels of CD90, CD63, CD34, and CD45 measured with western blotting; E. Expression of miR-221 in serum-derived exosomes from DPN and control rats; ${ }^{* * *} \mathrm{p}<0.001$. 
A

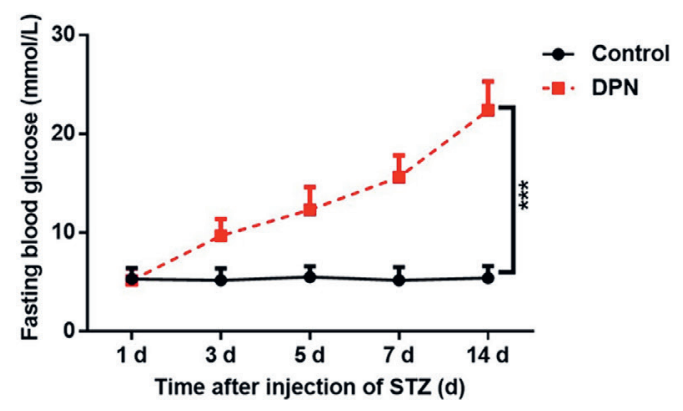

C

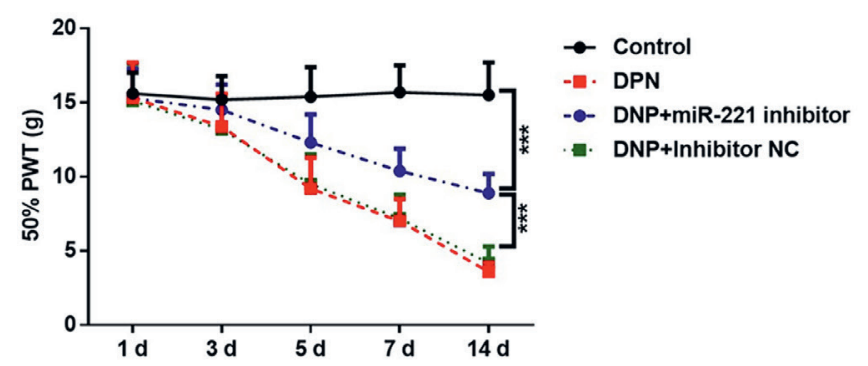

B
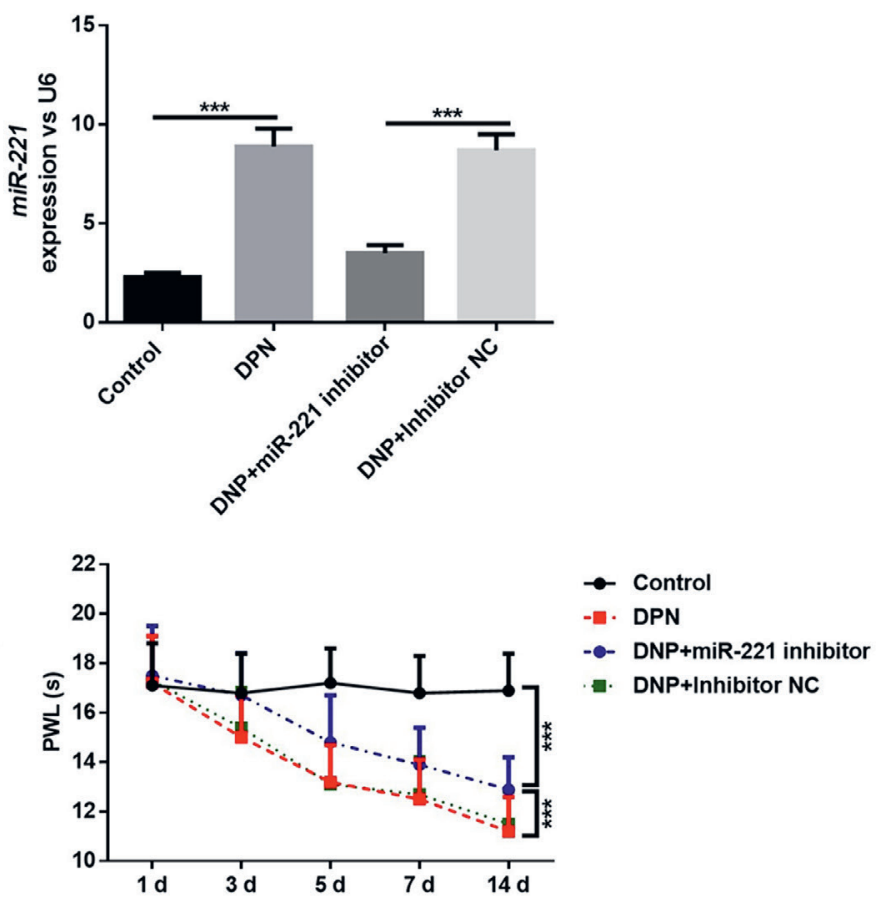

Fig. 2. Inhibition of miR-221 reduced the pain condition of STZ-induced DPN rats

A. Blood glucose levels for DPN and control rats; B. Expression of miR-221 in serum-derived exosomes from different groups of rats. C. 50\% PWT and PWL were evaluated in different groups of rats; ${ }^{* * *} \mathrm{p}<0.001$.

( $\mathrm{p}<0.05$, Fig. $2 \mathrm{C}$ ). When miR-221 was inhibited, both the $50 \%$ PWT and PWL values were significantly increased compared with the NC group $(\mathrm{p}<0.05)$. These results indicated that inhibition of $m i R-221$ improved the pain condition of DPN rats.

\section{Inhibition of miR-221 activated the expression of SOCS3 but suppressed the expression of NF-kB and inflammation factors in STZ-induced DPN rats}

To further reveal the effects of inhibiting $m i R-221$ in DPN, the serum protein levels of NF- $\mathrm{BB}$ and inflammation factors were evaluated. It was found that DPN treatment markedly decreased the expression of SOCS3 and increased the expression of NF- $\mathrm{kB}$ ( $\mathrm{p}<0.05$, Fig. 3A). However, knockdown of $m i R-221$ significantly reversed these effects. Furthermore, knockdown of $m i R-221$ remarkably decreased the expression of PEG2, BK, IL-6, IL-1 $\beta$, and TNF- $\alpha$ compared with the NC group, which was increased by STZ treatment in DPN rats ( $<0.05$, Fig. 3B). Taken together, these results indicate that knockdown of $m i R-221$ also decreased the expression of inflammation-related factors in DPN and that the downregulation of SOCS3 might be involved in this process.

\section{MiR-221 directly targeted and negatively regulated SOCS3}

Next, the binding mode between $m i R-221$ and SOCS3 was confirmed. The interaction between $m i R-221$ and SOCS3 was predicted using TargetScan v. 7.2 (http://www. targetscan.org/vert_72) and is shown in Fig. 4A. The dual luciferase reporter assay showed that luciferase activity significantly decreased when transfected with $m i R-221$ mimics and significantly increased when transfected with the miR-221 inhibitor in WT-SOCS3 ( $\mathrm{p}<0.05$ compared with the NC group, Fig. 3B). However, no significant difference was found in Mut-SOCS3. The mRNA and protein expressions of SOCS3 were also determined. As shown in Fig. 3C,D, inhibition of miR-221 markedly increased the expression of SOCS3, while overexpression of $m i R-221$ led to significant downregulation of SOCS3 compared with the NC group ( $<<0.05)$. These results indicate that miR-221 directly targeted and negatively regulated SOCS3.

\section{Inhibition of miR-221 suppressed inflammatory factors through regulation of SOCS3 in high glucose-induced SY5Y cells}

Lastly, miR-221 and SOCS3 were both suppressed in high glucose-induced SH-SY5Y cells and the expression of inflammatory factors was evaluated. It was found that the SOCS3 mRNA and protein expressions 


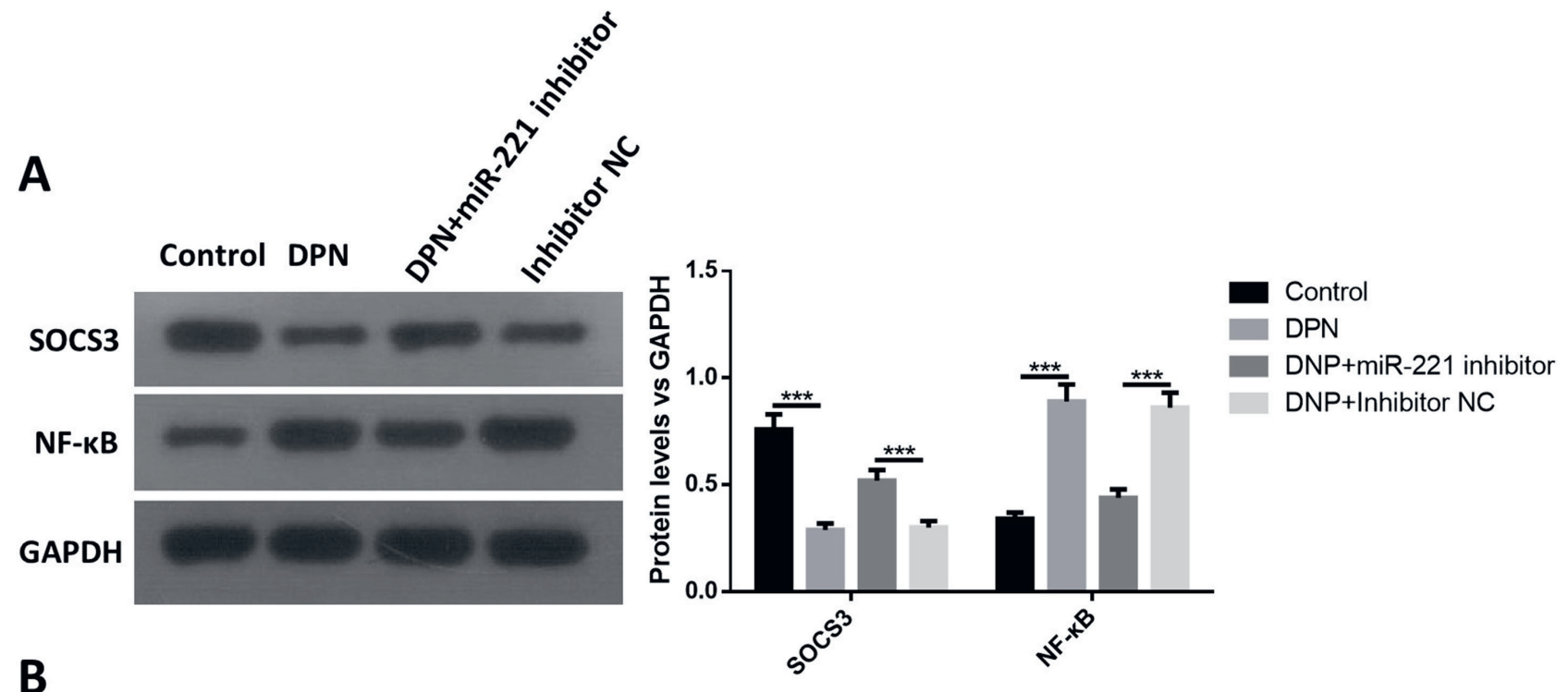

B

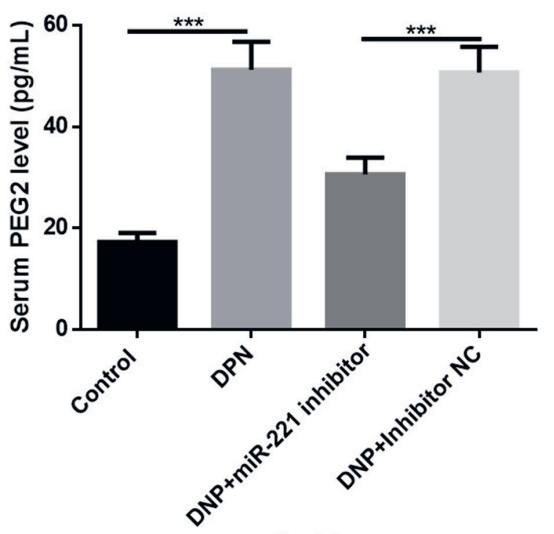

IL-1 $\beta$

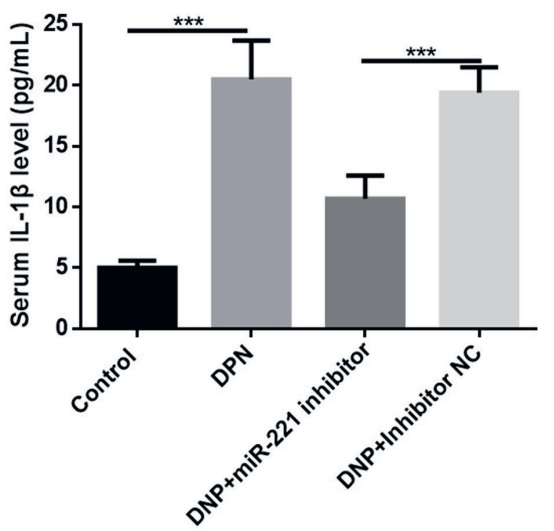

BK

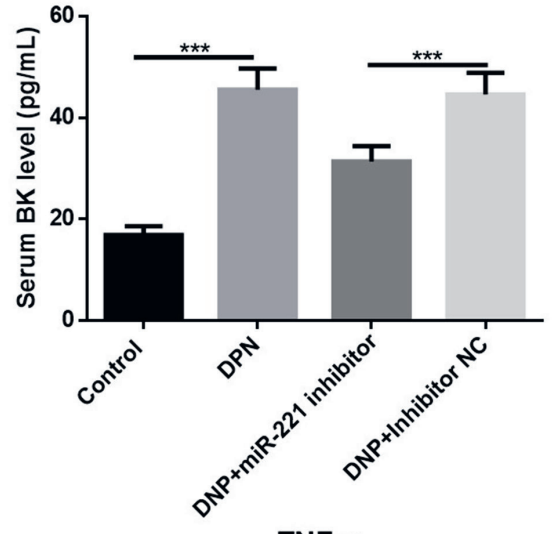

TNF- $\alpha$

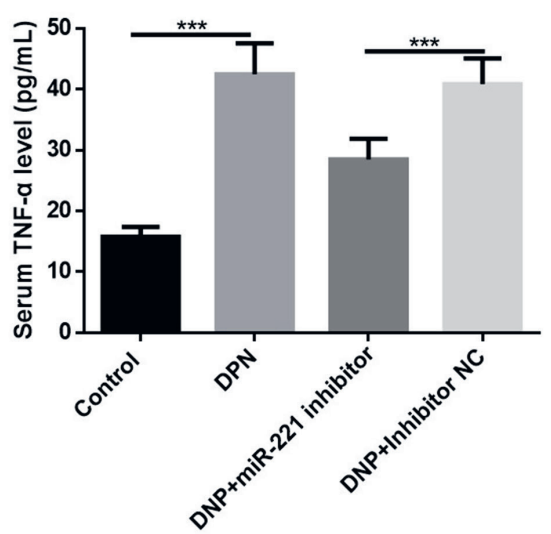

IL-6

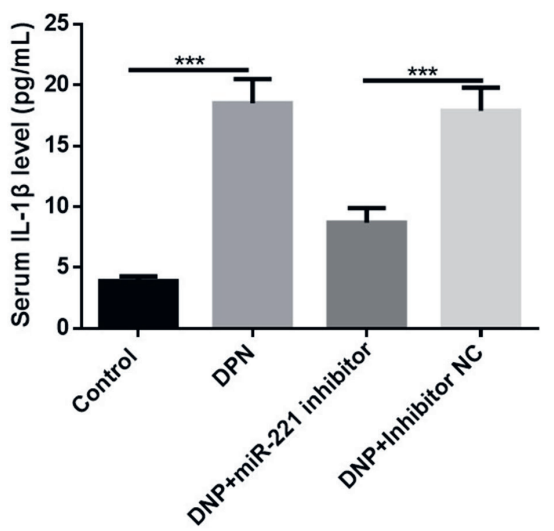

Fig. 3. Inhibition of miR-221 activated the expression of SOCS3 while suppressing the expression of NF-KB and inflammation factors in STZ-induced DPN rats A. Serum protein levels of SOCS3 and NF-KB in different groups of rats; B. Serum PEG2, BK, LL-6, IL-1 $\beta$, and TNF-a measured using ELISA; *** $p<0.001$.

were remarkably decreased in high glucose-induced cells compared with controls, while the expression of $m i R-221$ was remarkably upregulated in high glucose-induced cells ( $\mathrm{p}<0.05$, Fig. 5A,B). However, the suppression of $m i R-221$ markedly downregulated the expression of $m i R-221$ and upregulated the expression of SOCS3 in high glucose-induced cells $(\mathrm{p}<0.05)$. Co-transfection of the miR-221 inhibitor and si-SOCS3 significantly reversed the effects of the $m i R-221$ inhibitor. Furthermore, suppression of $m i R-221$ decreased the inflammatory levels of PEG2, BK, IL-6, IL-1 $\beta$, and TNF- $\alpha$, which were increased by high glucose. Inhibition of SOCS3 significantly reversed this effect (Fig. 5C). These results suggest that knockdown of $m i R-221$ suppressed high glucose-induced inflammatory factors through up-regulation of SOCS3. 
A
Position 1243-1250 of SOCS3 3' UTR
hsa-miR-222-3p
5' ...UGACAAUUUACAGGAAUGUAGCA... || $|\|| \mid$
3' UGGGUCAUCGGUCUACAUCGA

B

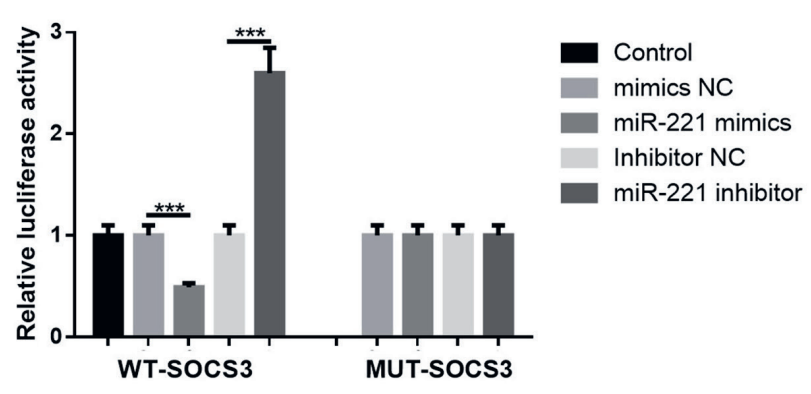

C
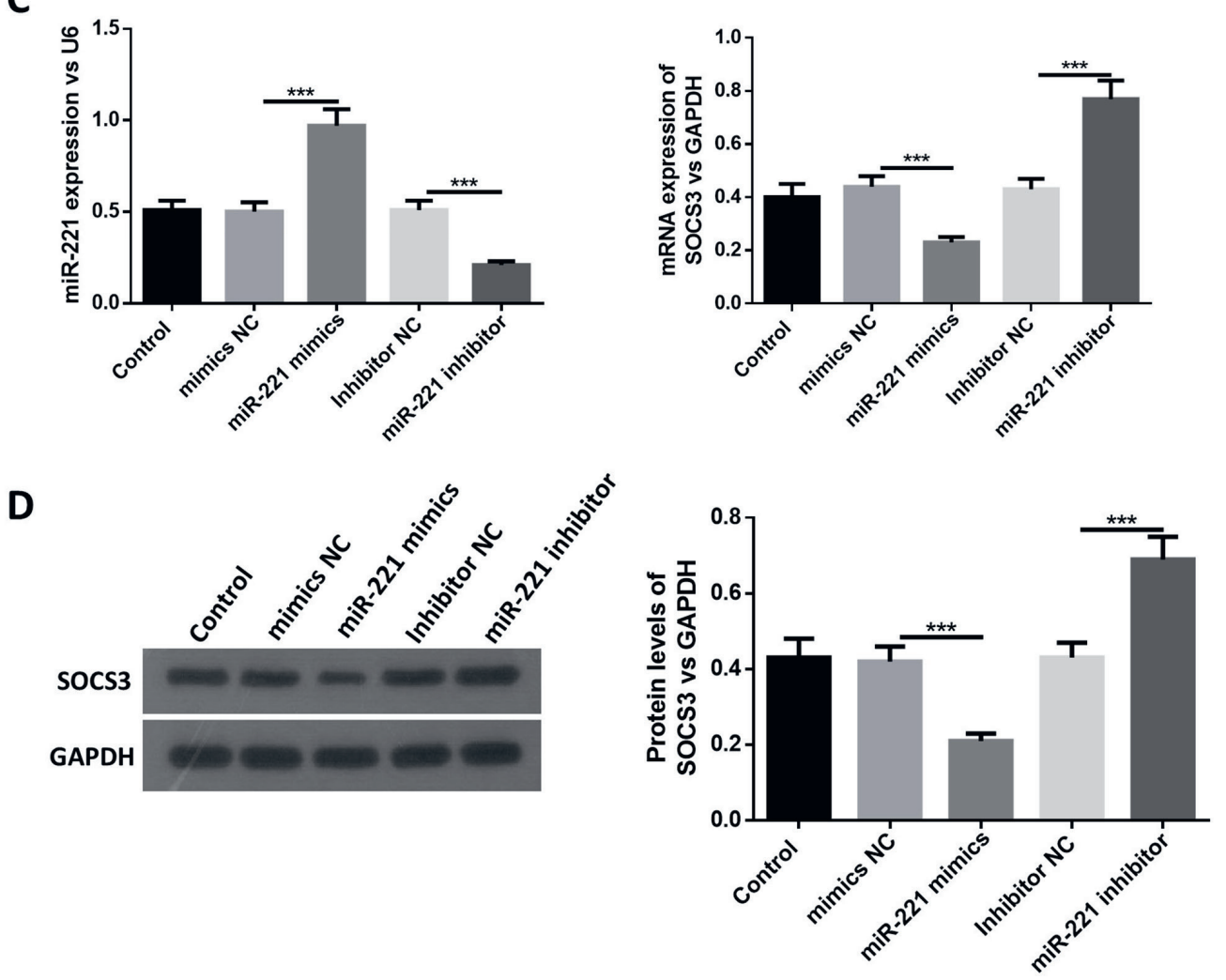

Fig. 4. MiR-221 directly targeted and negatively regulated SOCS3

A. Binding mode between miR-221 and SOCS3 predicted by TargetScan v. 7.2; B. Luciferase activity assessed with the dual luciferase reporter assay; C. Expression of miR-221 and SOCS3 in different groups of SY5Y cells measured using RT-qPCR; ${ }^{* *} p<0.001$.

\section{Discussion}

The DPN is one of the most common complications of diabetes. However, the molecular mechanisms of DPN remain unclear. In the present study, we demonstrated for the first time that $m i R-221$ is upregulated in DPN in vivo and in vitro models, and that inhibition of miR-221 improves DPN through targeting SOCS3.
The relationship between $m i R-221$ and diabetes has been noted in previous research. Lightell et al. demonstrated that $m i R-221$ and $m i R-222$ were upregulated in diabetes and that upregulation could promote intimal hyperplasia in diabetic mice. ${ }^{12}$ Qian et al. showed that inhibition of cell autophagy induced by $m i R-221$ resulted in aggravation of diabetic cardiac hypertrophy. ${ }^{13}$ All of these results suggest that $m i R-221$ is associated with aggravation of diabetes 

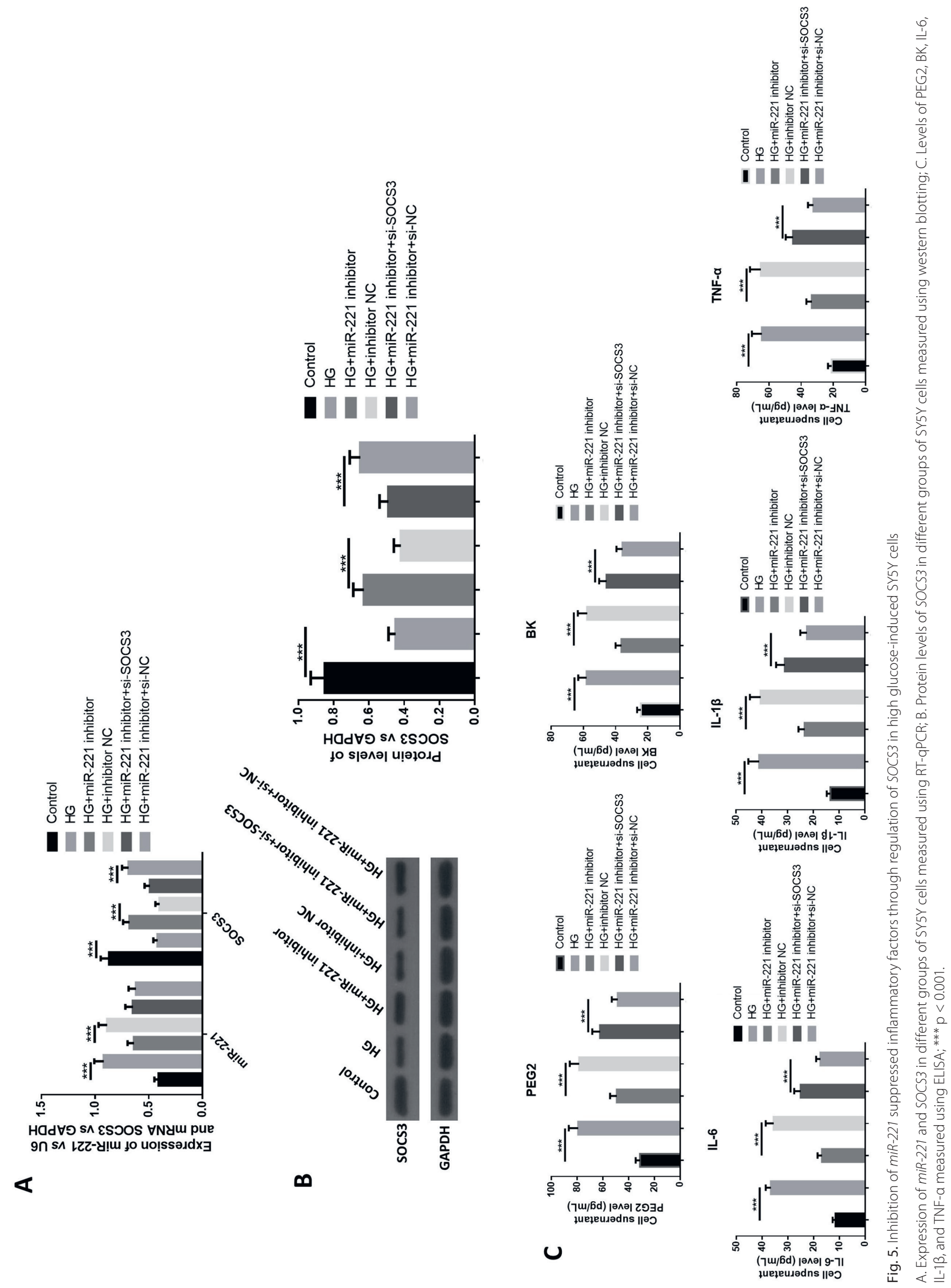
and its complications. In recent research, it was found that lncRNA GAS5 suppressed the proliferation and fibrosis of glomerular mesangial cells in diabetic nephropathy rats by targeting $m i R-221$ and negatively regulated its expression. ${ }^{14}$ In a clinical study, Liu et al. demonstrated that $m i R-221$ is also upregulated in diabetic retinopathy (DR) and has potential as a biomarker for DR. ${ }^{15}$ In a 2020 study, Fan et al. found that $m i R-221$ and $m i R-222$ could suppress the ability of islet cells to secrete insulin in a mouse model. ${ }^{16}$ These studies further indicate that $m i R-221$ plays a role as a promotor in diabetes. However, the role of $m i R-221$ in DPN has not been shown. In the present study, we confirmed that miR-221 was upregulated in a DPN rat model and that inhibition of $m i R-221$ improved the pain condition of DPN rats and inhibited inflammatory factors.

The relationships among $m i R-221$, inflammation and pain have been previously reported. It was found that $m i R-221$ can activate NF- $\mathrm{B}$ by inhibition of A20, and that NF- $\mathrm{BB}$ is a key factor in inducing inflammatory factors. ${ }^{17}$ Wang et al. demonstrated that inhibition of $m i R-221$ improved lipopolysaccharide (LPS)-induced inflammation in lung injury by suppressing SOCS1/NF-кB signaling. ${ }^{18}$ In a study of pain condition, Xia et al. found that inhibition of $m i R-221$ improved neuropathic pain by inhibition of suppressor of cytokine signaling $1 .{ }^{19}$ In our study, we also demonstrated that inhibition of $m i R-221$ improved pain condition in DPN rats and suppressed levels of inflammatory factors.

The SOCS3 is considered an inflammation suppressor and is also associated with diabetes. Research has shown that SOCS3 can suppress the expression of the inflammatory factors IL-1, IL-6 and tumor growth factor beta (TGF- $\beta$ ), as well as the inflammation signaling-related factors NF- $\mathrm{kB}$ and TGF $\beta .^{20,21}$ The SOCS 3 also shows antiinflammation effects in LPS-induced lung inflammation, hepatitis B virus (HBV)-induced inflammation, oxidative stress, and encephalomyelitis. ${ }^{22-24}$ Regarding the relationship between SOCS3 and diabetes, Zhu et al. showed that paeoniflorin could upregulate the expression of SOCS3 and inhibit inflammation, leading to suppression of matrix metalloproteinase-9 (MMP-9) and inflammatory factors in high glucose-induced renal clear cells. ${ }^{25}$ Duan et al. demonstrated that overexpression of SOCS3 improved high glucose-induced injury of pulmonary endothelial cells through inhibition of JAK2/STAT3 signaling. ${ }^{26}$ Another study observed that inhibition of IL-6 improved diabetic nephropathy and that the process was associated with upregulation of SOCS3. ${ }^{27}$ Taken together, these studies indicate that SOCS3 might improve diabetes, potentially through its anti-inflammation effects. In the present study, we showed that SOCS3 was downregulated in DPN rats and high glucose-induced cells, and that inhibition of SOCS3 reversed the beneficial effects of the $m i R-221$ inhibitor in high glucose-induced cells. The relationship between $m i R-221$ and SOCS3 has been reported in many other diseases, including prostate cancer, pancreatic cancer, thrombocytosis, and bladder cancer. ${ }^{28-30}$ However, this is the first confirmation that $m i R-221$ also targets SOCS3 in DPN.

\section{Limitations}

The present study has some limitations. The upstream molecules that regulate the $m i R-221 / S O C S 3$ axis are not sufficiently clear. Further research is also required to reveal the role of exosomes.

\section{Conclusions}

We demonstrated that inhibition of $m i R-221$ reduced pain and suppressed inflammatory factors in DPN rats, and also inhibited inflammation in a high glucose-induced SH-SY5Y cell line. This research might provide some novel molecular mechanisms for the development of DPN treatment.

\section{ORCID iDs}

Xiaole Wu (D) https://orcid.org/0000-0003-1002-309X Xiaoyu Wang (D) https://orcid.org/0000-0002-8699-4469 Yiyu Yin (D) https://orcid.org/0000-0002-3607-8622 Lei Zhu (1) https://orcid.org/0000-0001-8407-0388 Fengchao Zhang (1) https://orcid.org/0000-0001-7689-3122 Jianping Yang (D) https://orcid.org/0000-0001-9694-8899

\section{References}

1. Nathan CVS, Paul J, Abraham MM, Sasirekha M. Efficacy of low level laser therapy over conventional therapy on diabetic peripheral neuropathy: A pilot study. Call for Editorial Board Members. 2019;12(3):226.

2. Pop-Busui R, Boulton AJM, Feldman EL, et al. Diabetic neuropathy: A position statement by the American Diabetes Association. Diabetes Care. 2017;40(1):136-154. doi:10.2337/dc16-2042

3. Singh R, Kishore L, Kaur N. Diabetic peripheral neuropathy: Current perspective and future directions. Pharmacol Res. 2014;80:21-35. doi:10.1016/j.phrs.2013.12.005

4. Juster-Switlyk K, Smith AG. Updates in diabetic peripheral neuropathy. F1000Research. 2016;5:F1000 Faculty Rev-738. doi:10.12688/f1000 research.7898.1

5. Tesfaye S, Selvarajah D. Advances in the epidemiology, pathogenesis and management of diabetic peripheral neuropathy. Diabetes Metabol Res Rev. 2012;28:8-14. doi:10.1002/dmrr.2239

6. Dixit S, Maiya A, Shastry BA. Effect of moderate-intensity aerobic exercise on glycosylated haemoglobin among elderly patients with type 2 diabetes \& peripheral neuropathy. Indian J Med Res. 2017; 145(1):129-132. doi:10.4103/ijmr.IJMR_699_14

7. Teodoro JS, Nunes S, Rolo AP, Reis F, Palmeira CM. Therapeutic options targeting oxidative stress, mitochondrial dysfunction and inflammation to hinder the progression of vascular complications of diabetes. Front Physiol. 2019;9:1857. doi:10.3389/fphys.2018.01857

8. Sifuentes-Franco S, Pacheco-Moisés FP, Rodríguez-Carrizalez AD, Miranda-Díaz AG. The role of oxidative stress, mitochondrial function, and autophagy in diabetic polyneuropathy. J Diabetes Res. 2017; 2017:1673081. doi:10.1155/2017/1673081

9. Kozomara A, Birgaoanu M, Griffiths-Jones S. miRBase: From microRNA sequences to function. Nucleic Acids Res. 2019;47(D1):D155-D62. doi:10.1093/nar/gky1141

10. Oh SE, Park HJ, He L, Skibiel C, Junn E, Mouradian MM. The Parkinson's disease gene product DJ-1 modulates miR-221 to promote neuronal survival against oxidative stress. Redox Biol. 2018;19:62-73. doi:10. 1016/j.redox.2018.07.021 
11. Fornari F, Pollutri D, Patrizi C, et al. In hepatocellular carcinoma miR-221 modulates sorafenib resistance through inhibition of caspase-3-mediated apoptosis. Clin Cancer Res. 2017;23(14):3953-3965. doi:10.1158/ 1078-0432.CCR-16-1464

12. Lightell DJ, Moss SC, Woods TC. Upregulation of miR-221 and miR-222 in response to increased extracellular signal-regulated kinases $1 / 2$ activity exacerbates neointimal hyperplasia in diabetes mellitus. Atherosclerosis. 2018;269:71-78. doi:10.1016/j.atherosclerosis.2017. 12.016

13. Qian LB, Jiang SZ, Tang XQ, et al. Exacerbation of diabetic cardiac hypertrophy in OVE26 mice by angiotensin II is associated with JNK/CJun/miR-221-mediated autophagy inhibition. Oncotarget. 2017;8(63): 106661-106671. doi:10.18632/oncotarget.21302

14. Xiaoxu G, Bojin X, Wenwei X, Lili X, Shan H. Long noncoding RNA GAS5 inhibits cell proliferation and fibrosis in diabetic nephropathy by sponging miR-221 and modulating SIRT1 expression. Aging. 2019;11(20):8745-8759. doi:10.18632/aging.102249

15. Liu HN, Li X, Wu N, et al. Serum microRNA-221 as a biomarker for diabetic retinopathy in patients associated with type 2 diabetes. Int J Ophthalmol. 2018;11(12):1889-1894. doi:10.18240/ijo.2018.12.02

16. Fan L, Shan A, Su Y, et al. MiR-221/222 inhibit insulin production of pancreatic $\beta$-cells in mice. Endocrinology. 2019;161(1):bqz027. doi:10. 1210/endocr/bqz027

17. Zhao D, Zhuang N, Ding Y, Kang Y, Shi L. MiR-221 activates the NF-kB pathway by targeting A20. Biochem Biophys Res Commun. 2016;472(1): 11-18. doi:10.1016/j.bbrc.2015.11.009

18. Wang $T$, Jiang $L$, Wei $X$, et al. Inhibition of miR-221 alleviates LPSinduced acute lung injury via inactivation of SOCS1/NF-KB signaling pathway. Cell Cycle. 2019;18(16):1893-1907. doi:10.1080/15384101. 2019.1632136

19. Xia L, Zhang Y, Dong T. Inhibition of microRNA-221 alleviates neuropathic pain through targeting suppressor of cytokine signaling 1. J Mol Neurosci. 2016;59(3):411-420. doi:10.1007/s12031-016-0748-1

20. Babon JJ, Varghese LN, Nicola NA. Inhibition of IL- 6 family cytokines by SOCS3. Semin Immunol. 2014;26(1):13-19. doi:10.1016/j.smim.2013. 12.004
21. Gao A, Van DTE. Role of suppressors of cytokine signaling 3 in bone inflammatory responses. Front Immunol. 2013;4:506. doi:10.3389/ fimmu.2013.00506

22. Yan C, Ward PA, Wang X, Gao H. Myeloid depletion of SOCS3 enhances LPS-induced acute lung injury through CCAAT/enhancer binding protein $\delta$ pathway. FASEB J. 2013;27(8):2967-2976. doi:10.1096/fj.12-225797

23. Yuan K, Lei Y, Chen HN, et al. HBV-induced ROS accumulation promotes hepatocarcinogenesis through Snail-mediated epigenetic silencing of SOCS3. Cell Death Differ. 2016;23(4):616-627. doi:10.1038/ cdd.2015.129

24. Yan Z, Yang W, Parkitny L, et al. Deficiency of SOCS3 leads to braintargeted experimental autoimmune encephalomyelitis via enhanced neutrophil activation and ROS production. JCI Insight. 2019;4(9): e126520. doi:10.1172/jci.insight.126520

25. Zhu SH, Liu BQ, Hao MJ, et al. Paeoniflorin suppressed high glucoseinduced retinal microglia MMP-9 expression and inflammatory response via inhibition of TLR4/NF-KB pathway through upregulation of SOCS3 in diabetic retinopathy. Inflammation. 2017;40(5):1475-1486. doi:10.1007/s10753-017-0571-z

26. Duan WN, Xia ZY, Min L, Qian S, Yan L. Protective effects of SOCS3 overexpression in high glucose-induced lung epithelial cell injury through the JAK2/STAT3 pathway. MolMed Rep. 2017;16(3):2668-2874. doi:10.3892/mmr.2017.6941

27. Wu R, Liu X, Yin J, Wu H, Wang F. IL-6 receptor blockade ameliorates diabetic nephropathy via inhibiting inflammasome in mice. Metabolism. 2018;83:18-24. doi:10.1016/j.metabol.2018.01.002

28. Kneitz B, Krebs M, Kalogirou C, et al. Survival in patients with highrisk prostate cancer is predicted by miR-221, which regulates proliferation, apoptosis, and invasion of prostate cancer cells by inhibiting IRF2 and SOCS3. Cancer Res. 2014;74(9):2591-2603. doi:10.1158/00085472.CAN-13-1606

29. Liu B, Wu S, Ma J, et al. IncRNA GAS5 reverses EMT and tumor stem cell-mediated gemcitabine resistance and metastasis by targeting miR-221/SOCS3 in pancreatic cancer. Mol Ther Nucleic Acids. 2018;13: 472-482. doi:10.1016/j.omtn.2018.09.026

30. Navarro A, Pairet S, Alvarez-Larrán A, et al. MiR-203 and miR-221 regulate SOCS1 and SOCS3 in essential thrombocythemia. Blood Cancer J. 2016;6(3):e406. doi:10.1038/bcj.2016.10 